\title{
High-speed protein crystallography
}

\author{
The European XFEL produces its first protein structures.
}

A lmost 10 years ago, the first X-ray free-electron laser (XFEL) at the SLAC National Accelerator Laboratory in California ushered in a new approach to protein structure determination. This method, known as serial femtosecond crystallography, or SFX, captures single snapshots of individual protein microcrystals as they are streamed into a powerful laser firing femtosecondlength pulses. These diffraction patterns are indexed, merged, and interpreted to generate a $3 \mathrm{D}$ protein reconstruction. This approach has proven to be beneficial for studies of proteins that are difficult for traditional crystallography, including sensitive proteins that degrade upon radiation exposure, and structures such as membrane proteins, which often produce only small crystals.

Several other XFEL facilities have since been built around the world. However, none has been as hotly anticipated as the European XFEL (EuXFEL) facility in Hamburg, Germany, which opened to users last year. Now, two studies establish that high-resolution protein structures can be determined using this XFEL. One study was led by Ilme Schlichting and Thomas Barends of the Max Planck Institute for Medical Research, and Claudiu Stan of Rutgers University (Grünbein et al. 2018); the other was a large international collaboration led by Anton Barty and Henry Chapman of the Center for Free-Electron Laser Science (Wiedorn et al. 2018).

Both teams first solved the structure of a model protein, lysozyme, to demonstrate that reliable structural information can be measured at the EuXFEL. The researchers also determined structures of concanavalin A and B from a crude mixture of jack bean protein microcrystals (Grünbein et al. 2018), and of an unknown $\beta$-lactamase complex from Klebsiella pneumoniae (Wiedorn et al. 2018).

Despite both teams' considerable experience with XFEL-based

crystallography, at the EuXFEL, "everything was new," recalls Barty. Says Schlichting, "We spent a great deal of time planning and designing what we wanted to do." What makes the EuXFEL unique is its repetition rate-that is, the speed at which it fires femtosecond pulses, which is about 100 times faster than those of other XFELs.

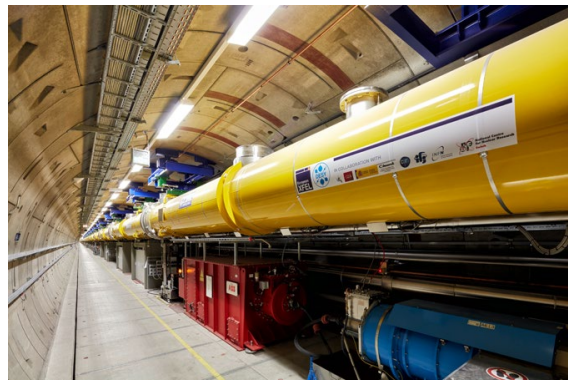

The EuXFEL accelerator tunnel. Credit: European XFEL/Heiner Müller-Elsner

Additionally, the pulses are produced in trains-that is, bursts of pulses with a megahertz firing rate interspersed with longer rest periods. Before these studies were undertaken, it was unclear how existing methods for sample delivery and data collection could be adapted to these novel features.

A common SFX sample-delivery method uses a liquid jet to introduce microcrystals in a narrow stream into the path of the laser beam. Each time a laser pulse hits the stream, it vaporizes the liquid, generating a gap; the stream must advance before the laser fires again. However, both groups found that high-speed liquid jets were fast enough for the high repetition rate of the EuXFEL.

But this was not the only challenge: as the laser hits the liquid stream, it causes a shock wave that can mechanically damage the unexposed crystals advancing toward the laser. Stray X-rays may also cause radiation damage to the advancing crystals. Both groups therefore carefully compared the diffraction data collected for the first pulse of each train, which should represent a pristine crystal, to those collected for subsequent pulses. Fortunately, when appropriate jetting conditions were used, damage to unexposed crystals was minimal.

A higher-speed detector than those used at other XFELs was also needed to record the megahertz-rate data. Both teams made use of a new ultrafast camera, the Adaptive Gain Integrating Pixel Detector (AGIPD), custom-designed for the EuXFEL.

Together, the studies establish that it is possible to harness the megahertz repetition rate of the EuXFEL to collect high-quality
SFX data in a much shorter amount of time, with lower sample consumption, than previously possible. Future upgrades to the EuXFEL should enable sufficient data for structure determination to be collected in just minutes, versus hours. Barty says that "this could be revolutionary," but both he and Schlichting point out that scheduling of experiments will need careful optimization to maximize users' time and avoid instrument downtime.

Other technical challenges and limitations remain. Further improvements in sample delivery are necessary; Barty highlights the need for more reliable liquid jet nozzles. He also notes that "the control, data collection and detector infrastructure need to be stress-tested to avoid unnecessary interruption to data collection." Schlichting notes that "due to the pulse pattern at the EuXFEL ... a significant part of the sample still goes to the drain." Finally, experiments at the EuXFEL will generate an enormous amount of data. Schlichting says that management of this deluge will require automated, reliable software for on-the-fly data sorting, as well as dedicated digital data banks.

These preliminary EuXFEL studies hint at exciting possibilities to come. Barty likens the technology development to the advance of flying over driving. "You can cover so much more ground in the same time that the rate of discovery is dramatically increased, and one can do things one would not have been able to do before," he says. The increased rate of data collection will enable users to perform better, more robust time-resolved crystallography studies to make molecular movies of enzyme mechanisms. With its unprecedented speed of data collection, the EuXFEL should also help open up the application of XFELs in structural biology to a broader community.

\section{Allison Doerr}

Published online: 30 October 2018 https://doi.org/10.1038/s41592-018-0205-X

\section{Research papers}

Grünbein, M. L. et al. Megahertz data collection from protein microcrystals at an X-ray free-electron laser. Nat. Commun. 9, 3487 (2018).

Wiedorn, M. O. et al. Megahertz serial

crystallography. Nat. Commun. 9, 4025 (2018). 